\title{
Crónica Comunitaria: actualidad institucional y económica de España en el marco de la Unión Europea (junio de 2018)
}

\author{
Communitarian Chronicle: Spanish up-to-date institutional \\ and economic framework within the European Union \\ (June 2018) \\ Beatriz Iñarritu \\ Profesora de la «Deusto Business School», Universidad de Deusto
}

doi: http://dx.doi.org/10.18543/ced-59-2018pp219-238

\begin{abstract}
Sumario: I. Introducción.-II. El Estado de la Integración. 1. Propuesta de profundización en la Unión Económica y Monetaria. 2. Brexit: Acuerdo sobre las condiciones del período transitorio del Brexit. 3. Luis de Guindos, vicepresidente del BCE.-III. Cuestiones generales de la actualidad económica. 1. BCE: Anuncio de la finalización del Programa de compras masivas de deuda (QE) en diciembre de 2018. 2. El Eurogrupo acuerda la finalización del rescate a Grecia y la reestructuración de la Deuda griega. 3. Guerra comercial con Estados Unidos ante la imposición de aranceles al acero y aluminio europeos. 4. MUR: polémica en relación a la resolución del Banco Popular. 5. Trabajadores desplazados: acuerdo sobre una nueva normativa. 6. Nuevas sentencias del Tribunal Europeo sobre las indemnizaciones a los trabajadores temporales españoles. 7. Semestre Europeo 2018: Informes en profundidad sobre Desequilibrios Macroeconómicos y Recomendaciones Específicas por país. 8. Competencia-Pacto fiscal Luxemburgo - Engie.
\end{abstract}

\section{Introducción}

Durante el primer semestre de 2018, la UE ha acordado con el Reino Unido la duración y las condiciones generales del periodo transitorio del Brexit, de manera que éste deberá hacerse realidad el 1 de enero de 2021. El gobierno británico también ha anunciado la regulación y el estatus que otorgará a los ciudadanos europeos que deseen continuar viviendo en el país.

También fueron noticias relevantes el anuncio del BCE de retirada de estímulos monetarios excepcionales a final de año y el nombramiento de 
Luis de Guindos como vicepresidente del Banco Central Europeo, una decisión que pretende contrarrestar la evidente infrarrepresentación española en las instituciones europeas.

El esperanzador acuerdo franco-alemán para avanzar en la Unión Económica y Monetaria y la revisión de la normativa sobre los trabajadores desplazados en la UE contrastaban con la preocupante guerra comercial iniciada contra Estados Unidos y con las dudas sobre el proceso de resolución del Banco Popular en 2017, que se derivan de la falta de transparencia en la información facilitada por la Junta Única de Resolución.

During the first six months of 2018, the European Union and the United Kingdom have agreed the duration and conditions of the Brexit transitional period, so that the withdrawal should be effective on 1 January 2021. The British government has also announced the regulation and status that will apply to the European citizens if they decide to continue to live in the country.

They were also remarkable those news about the end of the extraordinary monetary stimulus by the end of 2018 and the appointment of Luis de Guindos as vice-president of the European Central Bank, a decision that intends to counteract the evident lower representation of Spain in the European institutions.

The hopeful French-German pact on the new steps to be taken towards a deeper Economic and Monetary Union and the modification of the law about posted workers in the EU made the difference with the worrying commercial war with the United States and with the doubts about the resolution process of Banco Popular in 2017, derived from the lack of transparency in the information published by the Single Resolution Board.

\section{El estado de la integración}

\section{Propuesta de profundización en la Unión Económica y Monetaria}

Tras más de cuatro horas de reunión, la canciller alemana Angela Merkel y el presidente francés Emmanuel Macron hicieron público el pasado 19 de junio un acuerdo entre ambos países para crear un presupuesto común para la Eurozona que, en su caso, apoye iniciativas de inversión en innovación o en capital humano y que proteja a la región en épocas de fuertes crisis económicas.

Aunque evitaron hablar de cifras, sí anunciaron que este nuevo instrumento debería nutrirse con contribuciones nacionales y con futuros impuestos europeos como la tasa a las transacciones financieras, y una parte del Impuesto de Sociedades una vez que se armonicen las bases imponibles. 
El presupuesto de la Eurozona podría ponerse en marcha en 2021, en paralelo con el marco financiero plurianual de la UE y debería tener la convergencia económica entre países como objetivo, ya que en palabras de la canciller, «la convergencia en la Eurozona aún no es suficiente».

Respecto al Mecanismo Europeo de Estabilidad, MEDE, éste seguirá siendo el instrumento disponible para mejorar la estabilidad y afrontar problemas de liquidez de los Estados, evolucionando hacia un modelo de Fondo Monetario Europeo.

Por lo que se refiere a la Unión Bancaria, los líderes aludieron a la necesidad de avanzar en el tema del Fondo Europeo de Garantía de Depósitos bancarios para que, en efecto, los países con dificultades en sus sistemas financieros puedan contar con una red de seguridad común.

El acuerdo entre ambos líderes, que deberá ser ratificado por el Consejo Europeo de finales de junio, es clave para la profundización en la Unión Económica y Monetaria y, en todo caso, porque pone sobre la mesa la determinación del eje franco-alemán para encarar la oposición de un grupo de al menos ocho países, liderados por Holanda, que rechaza cualquier mutualización de riesgos o creación de nuevos fondos comunes.

\section{Brexit: Acuerdo sobre las condiciones del período transitorio del Brexit}

En diciembre de 2017 la UE de los Veintisiete y el Reino Unido hicieron público el acuerdo alcanzado sobre las condiciones de salida de los británicos de la UE, prevista para el 29 de marzo de 2019, y el negociador jefe de la UE, Michel Barnier, presentó la propuesta de la Comisión de que el periodo de transición del Brexit termine el 31 de diciembre de 2020, tres meses antes del plazo de dos años que había solicitado Londres, para hacerlo coincidir en el tiempo con la finalización del actual marco financiero plurianual de la UE.

En marzo se produjo el acuerdo sobre este período que, en efecto, retrasará 21 meses el Brexit definitivo y que fue presentado como un paso decisivo para evitar incertidumbres tanto para las empresas como para los 4,5 millones de ciudadanos europeos residentes en suelo británico y británicos en suelo europeo.

Durante este período Londres deberá cumplir la normativa comunitaria pero sin voz ni voto en la toma de decisiones, porque desde marzo de 2019, Reino Unido será, técnicamente, un país tercero para los socios comunitarios.

Las contrapartidas para Londres son difusas; la Unión concede que el Reino Unido pueda negociar y firmar acuerdos comerciales internacionales, aunque sólo podrían aplicarse una vez que finalice el período de transición.

Y respecto a Gibraltar, el acuerdo señalaba su inclusión en el período de transición, lo que significa que el peñón seguirá disfrutando de las ven- 
tajas del Mercado Único hasta finales de 2020. Pero también se especificaba en el pacto que su aplicación dependerá de la negociación bilateral entre España y Reino Unido, y para hacerla efectiva Madrid pretende que Londres haga concesiones en el uso compartido del aeropuerto gibraltareño, y en las prácticas fiscales agresivas del peñón.

El acuerdo sobre el «divorcio» daba pie al inicio formal de las negociaciones sobre la futura relación que vinculará a ambos bloques a partir del 1 de enero de 2021.

En junio, el Ministerio del Interior británico facilitó detalles importantes sobre el futuro de los ciudadanos de la UE que viven en Reino Unido tras el Brexit.

A partir del 29 de marzo de 2019 y hasta el 30 de junio de 2021, los europeos que lleven cinco años viviendo en el país tendrán que pagar 65 libras, dar su nombre y dirección y, además, demostrar que no tienen antecedentes penales graves para optar al «estatus de asentado» y poder permanecer de manera ilimitada en Reino Unido.

En cambio, los que hubieran llegado más recientemente, sin alcanzar los cinco años mencionados, deberán pedir la condición de «pre-asentados». Con este estatus podrán continuar residiendo en el Reino Unido hasta cumplir los cinco años, momento en el que podrán solicitar el estatus de «asentados».

Además, los familiares de estos ciudadanos europeos que vivan en otro país podrán viajar al Reino Unido incluso cuando termine el periodo de transición, siempre y cuando la relación de parentesco existiera antes del 31 de diciembre de 2020 y continúe existiendo una vez que esa persona se traslade al país. «Estamos demostrando un progreso real y espero con interés escuchar más detalles sobre cómo la UE hará arreglos recíprocos para los ciudadanos del Reino Unido que viven en la UE», afirmó la ministra británica de Inmigración, Caroline Nokes.

\section{Luis de Guindos, vicepresidente del BCE}

En febrero el gobierno español presentó la candidatura del entonces ministro de Economía Luis de Guindos a la vicepresidencia del Banco Central Europeo, responsabilidad que quedaba vacante el 31 de mayo al finalizar el mandato del portugués Vitor Constancio.

Fuentes del ejecutivo señalaron que el perfil de De Guindos era el de un candidato idóneo para el puesto, dado su papel fundamental en la gestión del ministerio de Economía y en la recuperación económica española y, en particular, en el saneamiento del sistema financiero y en el desarrollo de la Unión Bancaria. 
Sin embargo, a pesar de haber formado parte del Eurogrupo durante seis años como ministro español de Economia, esta experiencia también despertó cierto recelo en el seno del propio BCE, ya que podría poner en duda su independencia política. De Guindos no aportaba, además, un currículo como experto en política monetaria y su paso por el sector financiero estaba marcado por su trabajo en Lehman Brothers y en una de las cajas de ahorro nacionalizadas en España, Banca Mare Nostrum, BMN.

En cualquier caso, el Eurogrupo dio finalmente su apoyo al español el 19 de febrero, tras la retirada del otro candidato a ocupar el puesto, Philip Lane, gobernador del Banco Central de Irlanda. El apoyo expreso de un gran número de países (Alemania, Francia, Italia, Grecia, Eslovaquia, Malta y Portugal) hizo desistir a Irlanda sobre las opciones de su candidato.

La decisión fue ratificada un día después por los ministros de Economía y Finanzas de la Unión Europea. Y posteriormente, siguiendo el procedimiento previsto, también la comisión de Asuntos Económicos y Monetarios del Parlamento Europeo dio el visto bueno a su nombramiento el 27 de febrero.

El nombramiento oficial se produjo en el Consejo Europeo de los jefes de Estado y Gobierno de la UE celebrado en Bruselas el 22 de marzo, con lo que el español se convirtió en vicepresidente del BCE para un mandato no renovable de ocho años, a partir del 1 de junio.

\section{Cuestiones generales de la actualidad económica}

\section{BCE: Anuncio de la finalización del Programa de compras masivas de deuda $(Q E)$ en diciembre de 2018}

En junio el BCE anunció la retirada del programa de compras masivas de activos a finales de año. Desde octubre hasta diciembre de 2018 la intensidad de este «Quantitative Easing», QE, bajará hasta 15.000 millones de euros mensuales, siguiendo la senda ya iniciada a comienzos de año de reducir la compra a 30.000 millones al mes entre enero y septiembre.

A partir de diciembre el BCE dará por terminado este programa excepcional a no ser que, en efecto, la inflación u otros indicadores macroeconómicos pudieran obligar a revertir la decisión.

La inflación parece recuperarse en la Eurozona gracias al alza del precio del petróleo, pero también es cierto que el incremento de los precios podría hacer mella en la recuperación, lo que genera cierta incertidumbre respecto al futuro.

Aunque la autoridad monetaria de la Eurozona anunció que los tipos de interés se mantenían intactos en su mínimo histórico del $0 \%$ en vigor 
desde marzo de 2016, dio una primera señal sobre cuándo podría llegar una primera subida al afirmar que este mínimo actual se mantendrá invariable hasta al menos el verano de 2019.

A pesar de que, de esta forma, el BCE comenzó a poner ciertas fechas a su repliegue de estímulos, la institución no quiso transmitir ningún mensaje concreto sobre una inminente subida de tipos. De hecho, Mario Draghi recordó en rueda de prensa que la política monetaria seguiría siendo acomodaticia, con los tipos al $0 \%$ y con la extensión de las compras de deuda hasta el mes de diciembre de 2018.

\section{El Eurogrupo acuerda la finalización del rescate a Grecia y la reestructuración de la Deuda griega}

Los ministros de Finanzas del euro cerraron el 21 de junio un acuerdo para finalizar el último de los rescates a Grecia sobre la base de nuevas condiciones para reestructurar el pago de su deuda.

Tras tres programas de asistencia financiera al país, que han acumulado un total de 273.000 millones de euros en préstamos por parte de sus socios de la Eurozona, los ministros daban por finalizado un período crítico en la historia reciente del país que debería permitir a Atenas acceder a los mercados financieros el 20 de agosto.

El país ha debido soportar desde 2010, cuando se pactó el primer rescate, una profunda intervención por parte de las instituciones de la Eurozona, con fuertes medidas de austeridad que han resultado en una reducción del $25 \%$ en su PIB, una disminución del $40 \%$ en los ingresos de los hogares y la pérdida de un millón de empleos. El país debió pactar una quita de su deuda en 2012, convocó un referéndum sobre el tercer rescate y sufrió un corralito bancario en el verano de 2015 .

Este nuevo acuerdo sólo fue posible una vez que los miembros del Eurogrupo confirmaron que Atenas ha cumplido las medidas prioritarias exigidas y que los esfuerzos realizados han sido suficientes y tras certificar su confianza en la promesa de Grecia de mantener el déficit primario en el 3,5\%.

Sin embargo, no fue un acuerdo fácil, puesto que durante más de nueve horas de reunión, los ministros escenificaron las fuertes divergencias existentes entre países como Alemania y Holanda, partidarios de ofrecer condiciones menos generosas sobre la reestructuración de la deuda griega y otros países liderados por Francia más alineados del lado de Grecia que reclamaban más solidaridad con el país.

Finalmente, el pacto alcanzado sobre la reestructuración de la deuda griega plantea una extensión del plazo de carencia de 10 años para el comienzo del pago de capital e intereses del tercer rescate, una ampliación se- 
mejante de otros diez años para su vencimiento y la entrega de un último tramo de 15.000 millones de euros.

El Eurogrupo, además, se declaraba dispuesto a considerar nuevas medidas de reestructuración de la deuda griega en caso de que «se materialicen escenarios económicos adversos».

Con la decisión del Eurogrupo, Atenas recuperaba por fin las riendas de su soberanía en materia de política económica, aunque «la troika», formada por representantes de la Comisión Europea, Banco Central Europeo y Fondo Monetario Internacional, seguirá visitando el país para comprobar que las reformas no se revierten y para supervisar la situación fiscal.

Esta supervisión es obligatoria hasta que el país rescatado por la Eurozona devuelva, al menos, el 70\% del préstamo, por lo que habida cuenta del enorme paquete de deuda en manos del MEDE (organismo de la Eurozona para articular los rescates a Estados en dificultades) y la extensión en el vencimiento acordada, es más que previsible que las misiones de los «hombres de negro» continúen durante mucho tiempo, quizás durante las próximas dos décadas. Y, lógicamente, no es descartable que con esta supervisión se pudiera obligar a Grecia a bloquear políticas o exigir cambios en materia de política económica, si se perciben desviaciones o incumplimientos en las medidas comprometidas.

A pesar de que, en efecto, Grecia continuará bajo vigilancia, los mensajes de optimismo se sucedieron tras la decisión del Eurogrupo, en la medida en que muchos medios la interpretaron como el símbolo del fin de la crisis del euro. El presidente de esta reunión de ministros de finanzas, Mário Centeno, señaló que Grecia había completado con éxito el programa tras aplicar centenares de medidas, «a menudo con mucha dureza para la población». Y no olvidó, ante lo que consideró la apertura de una nueva etapa, rendir un merecido tributo al pueblo griego, por «su resiliencia y compromiso europeo». «La crisis griega acaba aquí en Luxemburgo esta noche», señaló también el comisario de Asuntos Económicos, Pierre Moscovici.

En todo caso, lo cierto es que Atenas «recuperará su libertad» el 20 de agosto con una deuda que supera el $178 \%$ de su PIB, por lo que las incertidumbres sobre su capacidad para hacerla frente son obvias, incluso si el país cumpliese estrictamente las reformas aprobadas durante estos años.

Tanto el FMI como el BCE se hicieron eco de estas dudas, y llegaron a plantearlas públicamente. El presidente del BCE, Mario Draghi, señaló, en este sentido, que «damos la bienvenida a la disposición del Eurogrupo a considerar más medidas de alivio a largo plazo si se materializaran desarrollos económicos adversos», lo que fue interpretado como una señal del te- 
mor del Banco a que, en unos años, tuviera que volver a abordarse la cuestión de la deuda griega.

Estas tesis contrastan con las mostradas por las instituciones europeas, ya que éstas han basado su confianza en la capacidad de Grecia para asumir los pagos de su deuda en la propia composición de la deuda, con largos vencimientos, bajos intereses y en una proporción mayoritaria en manos de ellas mismas, lo que podría hacer el calendario de pagos más soportable.

\section{Guerra comercial con Estados Unidos ante la imposición de aranceles al acero y aluminio europeos}

Estados Unidos decidió el 1 de junio iniciar una guerra comercial contra sus grandes aliados, la Unión Europea, México y Canadá, al activar unos aranceles al acero (25\%) y al aluminio (10\%) que había anunciado en marzo pero dejado en suspenso con el fin de abrir una eventual negociación.

Washington, que pedía cambios para reducir el déficit comercial con dichos socios comerciales consideró no haber conseguido una solución satisfactoria durante las conversaciones y por esta razón anunció el 31 de mayo que los gravámenes entraban en vigor en la medianoche de ese mismo día.

El gobierno de Donald Trump ha defendido desde su llegada al poder que el déficit comercial del país es el gran mal del sector industrial estadounidense y, por tanto, origen de todos los males de la clase media. Así es como se explica que Washington haya iniciado una guerra comercial contra sus más importantes socios, Europa, Canadá, México y China.

Según declaró el secretario de Estado de comercio de Estados Unidos, Wilbur Ross, las conversaciones con los socios europeos habían logrado «algunos avances», pero no los suficientes para mantener la exención a los nuevos aranceles, que expiraba en la medianoche del 31 de mayo.

Según expertos en la materia, la intención de Estados Unidos durante las conversaciones fue tratar de cerrar acuerdos con medidas que, a la postre, establecieran limitaciones voluntarias en las exportaciones al país. Es el tipo de pactos que, en efecto, ha ido consiguiendo con Corea del Sur, Australia, Brasil y Argentina, que sí mantienen la exención sobre las tarifas en sus exportaciones.

«Hoy es un mal día para el comercio mundial. Hemos hecho todo lo posible para evitar este resultado», lamentó la comisaria de Comercio, Cecilia Malmström. «En estas conversaciones, Estados Unidos ha tratado de usar la amenaza de restricciones comerciales para obtener concesiones de la UE. Nosotros no negociamos así», reprochó. 
Bruselas anunció el mismo 1 de junio que ese mismo día denunciaría a Estados Unidos ante la Organización Mundial del Comercio, OMC, y que impondría «medidas de reequilibrio» para proteger a su industria.

«Es puro y simple proteccionismo. Estados Unidos no nos deja otra opción que proceder a presentar el caso ante la OMC e imponer más aranceles a una serie de importaciones de EE.UU.», anunció el presidente de la Comisión Europea, Jean-Claude Juncker, en un comunicado.

De hecho, cuando Estados Unidos anunció su primera amenaza contra el acero y el aluminio europeos, Bruselas elaboró una lista de unos 350 productos (que incluye una gran variedad de bienes, como alimentos, bebidas, maquillaje o barcos de recreo) que quedarían sujetos a gravámenes adicionales como represalia por las medidas estadounidenses. La Comisión Europea la notificó a la OMC en mayo para poder actuar de manera inmediata si Washington decidía finalmente aplicar los aranceles al acero y aluminio de la UE.

En concreto Bruselas presentó dos listados de productos estadounidenses que eventualmente serían penalizados y cuyas ventas a la UE representan unos 6.400 millones de euros anuales, un montante equivalente a las ventas europeas de acero y aluminio a Estados Unidos, en una búsqueda de equiparación de daños.

Este fue el antecedente de la decisión anunciada por la Comisión el 20 de junio sobre la aplicación, a partir del día 22, de unos aranceles del $25 \%$ a una lista de productos estadounidenses, entre los que se incluyen productos simbólicos e icónicos del país como la mantequilla de cacahuete, los vaqueros Levi's, el whisky Bourbon, el maíz dulce, los arándanos o el zumo de naranja. En total, se verán afectados por esta primera oleada de aranceles casi 200 productos cuyo valor de importación en la UE asciende a 2.800 millones de euros.

«Nuestra respuesta es medida, proporcionada y está plenamente en línea con las reglas de la OMC», señaló la comisaria Malmström, recordando asimismo que si Estados Unidos diera marcha atrás y eliminara los aranceles sobre el acero y aluminio europeos, Europa también retiraría los suyos.

Al margen de estas represalias, sobre una suma estimada de importaciones de 2.800 millones de euros, la UE se reserva la posibilidad de imponer una segunda tanda de aranceles sobre otros 3.600 millones de euros en importaciones procedentes de Estados Unidos, una vez que hubieran transcurrido tres años desde el anuncio de las medidas estadounidenses, es decir, en marzo de 2021, o antes si la OMC resolviera la disputa contra Estados Unidos y a favor de la UE. Con esta segunda imposición de aranceles, la Unión podría equiparar la cifra de 6.400 millones relativa a la venta del acero y del aluminio europeos en Estados Unidos.

Este conflicto comercial con EE.UU. se añadía al anuncio de la imposición de aranceles a la aceituna negra española, un sector con gran impor- 
tancia en Andalucía, formado por casi 300 empresas y que suma unos 8.000 empleos.

Washington culpaba directamente a las subvenciones que los agricultores andaluces reciben de la UE, que hacen posible su venta por debajo de su valor de mercado, para ratificar y aumentar el arancel desde el 21,6\% provisional establecido en los meses de noviembre y enero hasta el $35,75 \%$ definitivo establecido en junio.

Fuentes de la Comisión Europea calificaron las tasas de inadmisibles e injustificadas y anunciaron que se tomarían medidas de manera inmediata. De hecho, será preciso esperar a la reunión de la Comisión de Comercio Internacional, prevista para el 24 de julio, para conocer la decisión de este organismo sobre su conformidad o su rechazo respecto de dichos aranceles.

\section{MUR: polémica en relación a la resolución del Banco Popular}

En junio de 2017 se produjo la primera actuación del Mecanismo Único de Resolución (MUR) de la Eurozona. El riesgo de colapso por el hecho de que el Banco Popular no hubiera podido atender las retiradas de dinero de sus clientes llevó a las autoridades europeas a decidir, por vez primera, una intervención rápida e inédita de «resolución» de un banco europeo.

Tras el preceptivo dictamen del Banco Central Europeo en el que se planteaba la necesidad de dicha intervención ante la delicada situación del Popular, que estaba a punto de caer o era probable que cayese («failing or likely to fail»), la Junta Única de Resolución tomó la decisión de encargar al Fondo de Reestructuración Ordenada Bancaria, el FROB español, la venta del banco, operación que finalmente se cerró con el Banco Santander, quien aceptó la compra del Popular por la simbólica cifra de un euro.

Sin embargo, la operación provocó una fuerte polémica habida cuenta del perjuicio causado a unos 300.000 accionistas y poseedores de deuda del banco que, de un día para otro, perdieron toda su inversión.

En poco tiempo se produjo un alud de demandas ante el Tribunal de Justicia de la UE contra la JUR y ante la Audiencia Nacional contra el FROB, además de querellas contra las ex cúpulas de la entidad bancaria. Por la vía civil también los inversores iniciaron miles de pleitos contra el Popular y el Santander en los juzgados de toda España.

Ante la falta de transparencia del proceso de venta de la entidad española, el Panel de recurso de la JUR decidió el 28 de noviembre de 2017 que una serie de versiones no confidenciales de determinados informes clave para la resolución del banco debían hacerse públicos, decisión que finalmente fue acatada a comienzos de febrero de 2018. 
Aunque la JUR evitó la publicación de determinados datos considerados sensibles, uno de los informes, elaborado por la propia Junta, despertó especial interés, ya que desvelaba que apenas 48 horas antes de la decisión de resolución del banco, este organismo consideraba que la valoración provisional no indicaba que el banco fuera insolvente o inviable. Esta consideración significaba, por tanto, que la resolución de la entidad se fundamentó no tanto en la insolvencia sino en una grave crisis de liquidez y de financiación, provocada por una retirada masiva de depósitos bancarios de sus clientes.

Y otro informe sobre la valoración económica del Popular, elaborado por la auditoría Deloitte y que justificó la venta del banco al Santander, aportaba un rango de valoración del banco de entre $-8,000$ millones de euros en un escenario pesimista y 1.300 millones en otro optimista. En el escenario base el valor de la entidad se situaba en -2.000 millones, siendo esta última cifra la referencia final aplicada por la JUR.

Sin embargo, representantes legales de los inversores afectados pusieron en duda estas valoraciones y señalaron que únicamente podrían haber sido válidas si los activos del banco se hubieran valorado como si su venta fuera urgente y no en un proceso de venta ordenado. La JUR, por su parte, señalaba que la valoración debía tener en cuenta los potenciales riesgos y los costes que debería asumir el comprador de la entidad, incluidos los riesgos legales y costes derivados de las demandas contra los gestores del Popular. El Santander ofreció 1 euro y dado que el valor en el escenario base se situó en -2.000 millones, el precio se consideró adecuado.

En su defensa Deloitte afirmaba que tuvo un tiempo «extremadamente corto» para elaborar el informe, poco más de diez días, cuando había pactado con la JUR contar con «al menos seis semanas». Señaló, además, que no tuvo acceso a «cierta información crítica» y que tuvo «oportunidades limitadas» de debatir con expertos y afectados.

Reconocía de esta forma que su informe incluía una valoración «altamente incierta y provisional» y que añadía un «colchón» añadido para posibles pérdidas adicionales imposibles de estimar entonces. Y fuentes de la JUR admitieron, tras la publicación de los documentos, que la intervención se llevó a cabo de manera precipitada y con exceso de urgencia.

Las reclamaciones de los inversores afectados no cesaron ya que calificaron de insuficiente la información facilitada por la JUR, por lo que volvieron a recurrir al Panel. Y en junio consiguieron una segunda decisión de este organismo para forzar la publicación de nueva información sobre el proceso de resolución del Popular.

La JUR deberá desvelar información sobre la fuga de depósitos diaria, de mayo y junio de 2017, y datos sobre la liquidez de la entidad bancaria, y 
deberá también publicar datos clave del informe de evaluación de Deloitte y otros documentos que no había publicado en febrero. El Panel justificaba esta decisión porque sin estos datos el informe de la auditora es «casi ininteligible» y hace «imposible para los afectados y para los tribunales comprender su rol en el informe».

\section{Trabajadores desplazados: acuerdo sobre una nueva normativa}

En marzo las instituciones europeas alcanzaron un principio de acuerdo para modificar la Directiva comunitaria sobre trabajadores desplazados en el marco de una prestación de servicios e introducir su equiparación salarial con los trabajadores locales, lo que podría suponer una mayor retribución para más de dos millones de empleados.

El texto acordado fijaba un periodo máximo del desplazamiento de 12 meses, con posibilidad de establecer una prórroga justificada de seis meses adicionales. Transcurrido ese plazo, el trabajador podrá acogerse a la legislación laboral del país de destino si es más favorable que la de su país de origen.

La Directiva anterior al acuerdo fue aprobada en 1996 y solo exige una remuneración igual al salario mínimo del país a donde se desplaza el trabajador, lo que genera situaciones de dumping laboral en sectores con gran movilidad laboral como la construcción. La norma anterior también ha facilitado el desplazamiento cuasi permanente de trabajadores procedentes de países más pobres hacia otros más prósperos, recibiendo unos salarios muy inferiores a los habituales en el país de destino. Entre 2010 y 2014, el número de empleados desplazados aumentó en un $40 \%$ hasta casi dos millones de trabajadores.

Esta nueva directiva era una prioridad declarada por el presidente de la Comisión Europea, Jean-Claude Juncker, a la vista del incremento en el número de trabajadores desplazados registrado durante la crisis y contaba con el apoyo de países como Francia, Alemania, Italia, Holanda o Bélgica, partidarios de endurecer el control de los trabajadores desplazados a sus territorios, y de combatir el dumping salarial.

Entre 2010 y 2015, España dobló el número de trabajadores desplazados a otros países de la UE mientras que Polonia, país que lidera la exportación de trabajadores, la incrementó en un 74\%, hasta los 463.000 trabajadores. El 23\% de todos los trabajadores desplazados en Europa son polacos.

El Parlamento Europeo aprobó la nueva Directiva en su sesión plenaria del 29 de mayo con 456 votos a favor, 147 en contra y 49 abstenciones. Su contenido deberá hacerse efectivo en los Estados miembros en un plazo de 
dos años, ya que éstos dispondrán de este plazo, desde su aprobación formal, para transponerla a su legislación nacional.

Se trata de una norma que, sin duda, mejorará la protección de los trabajadores desplazados y que asegurará unas condiciones de competencia más justas para las empresas.

\section{Nuevas sentencias del Tribunal Europeo sobre las indemnizaciones a los trabajadores temporales españoles}

El 5 de junio el Tribunal de Justicia de la Unión Europea hizo públicos dos fallos que han contribuido a clarificar el hecho de que la normativa laboral española no es discriminatoria en el trato a los trabajadores fijos y temporales y que, por tanto, es posible establecer indemnizaciones diferentes para ambas tipologías de contratos.

Los jueces europeos dictaminaron que, en efecto, fijos y temporales no necesariamente deben tener derecho a la misma indemnización

Y, en particular, en uno de los fallos el Tribunal señalaba que el acuerdo marco europeo sobre trabajo de duración determinada «no se opone» a una normativa nacional, la española en este caso, que prevé diferentes indemnizaciones y que, en particular, no prevé el abono de indemnización alguna a los trabajadores interinos.

De esta forma, la Corte europea rectificaba un pronunciamiento suyo anterior de Septiembre de 2016, cuando dictó una polémica y confusa sentencia en la que podía interpretarse que un trabajador temporal interino que, en efecto, según la ley española no tiene derecho a indemnización, debería recibir el mismo trato que un trabajador indefinido despedido por causas objetivas (20 días por año).

Sin embargo, casi dos años después, el argumento aportado por los jueces europeos contradecía esta primera tesis, al afirmar que en el caso de un trabajador temporal interino las partes del contrato conocen desde el principio la fecha o acto que causará el fin de la relación laboral mientras que en el caso de un trabajador indefinido que es despedido por causas objetivas a iniciativa del empresario, corresponde una indemnización para compensar el carácter imprevisto de la ruptura de la relación laboral.

Expertos laboralistas expresaron su opinión de que, efectivamente, estas nuevas sentencias rectificaban el pronunciamiento anterior de la Corte porque en esta ocasión los jueces justificaban que la posición de un trabajador indefinido que es despedido es distinta a la de un temporal. Los trabajadores indefinidos no saben cuándo se va a terminar la relación laboral y el daño, por tanto, es mayor. 
Y también señalaron estos expertos que las sentencias dejaban la puerta abierta a la posibilidad de equiparar a los interinos con el resto de contratos temporales, es decir, a la posibilidad de establecer una indemnización similar para este colectivo de trabajadores interinos.

\section{Semestre Europeo 2018: Informes en profundidad sobre Desequilibrios Macroeconómicos y Recomendaciones Específicas por país}

La Comisión Europea presentó en febrero el llamado «Paquete de Invierno» que integra los diferentes Informes-país que, en su caso, incluyen los Exámenes Exhaustivos derivados del Informe del Mecanismo de Alerta, IMA, publicado en noviembre.

La Comisión certificaba entonces que la Economía europea continuaba creciendo con fuerza y que las perspectivas económicas positivas se veían acompañadas por una mejoría en el mercado laboral y en la situación social. Sin embargo, también alertaba sobre el hecho de que la recuperación no parecía estar beneficiando a toda la sociedad de forma equitativa y que las debilidades estructurales estaban frenando el crecimiento y la convergencia en algunos Estados miembros.

Los 27 informes por país presentados, para todos los Estados miembros excepto Grecia que aún entonces era objeto de un programa específico de apoyo financiero, incluían el análisis anual, por parte de los servicios de la Comisión de su situación económica y social, así como de los avances en la aplicación a lo largo de los años de las Recomendaciones Específicas por País, REPs. Para los 12 Estados miembros seleccionados en noviembre en el IMA, los informes por país incluían sus respectivos Exámenes Exhaustivos, con una evaluación particular de los posibles desequilibrios macroeconómicos que actualizaba la categorización de los países en el marco del denominado «Procedimiento de Desequilibrios Macroeconómicos».

En términos generales, la Comisión certificaba que los desequilibrios se están corrigiendo gracias a las reformas en curso y a la recuperación económica, y es por ello que el número de países vigilados por este procedimiento está disminuyendo. Destacaba, en particular, los progresos alcanzados en Bulgaria, Eslovenia, Francia y Portugal, con un cambio positivo de categoría.

En efecto, la conclusión respecto a la actualización de la clasificación de países tras la publicación de los Exámenes Exhaustivos fue la siguiente:

- Eslovenia ya no sufre desequilibrios económicos, 
- Alemania, Bulgaria, España, Francia, Irlanda, los Países Bajos, Portugal y Suecia muestran desequilibrios económicos, destacando el hecho de que Bulgaria, Francia y Portugal mejoraban respecto a la clasificación de 2017,

- Chipre, Croacia e Italia continúan con desequilibrios económicos excesivos.

El comisario Pierre Moscovici, responsable de Asuntos Económicos y Financieros, Fiscalidad y Aduanas, afirmó, con motivo de la publicación de los informes, que once países continuaban experimentando desequilibrios «que los hacen vulnerables en caso de perturbaciones».

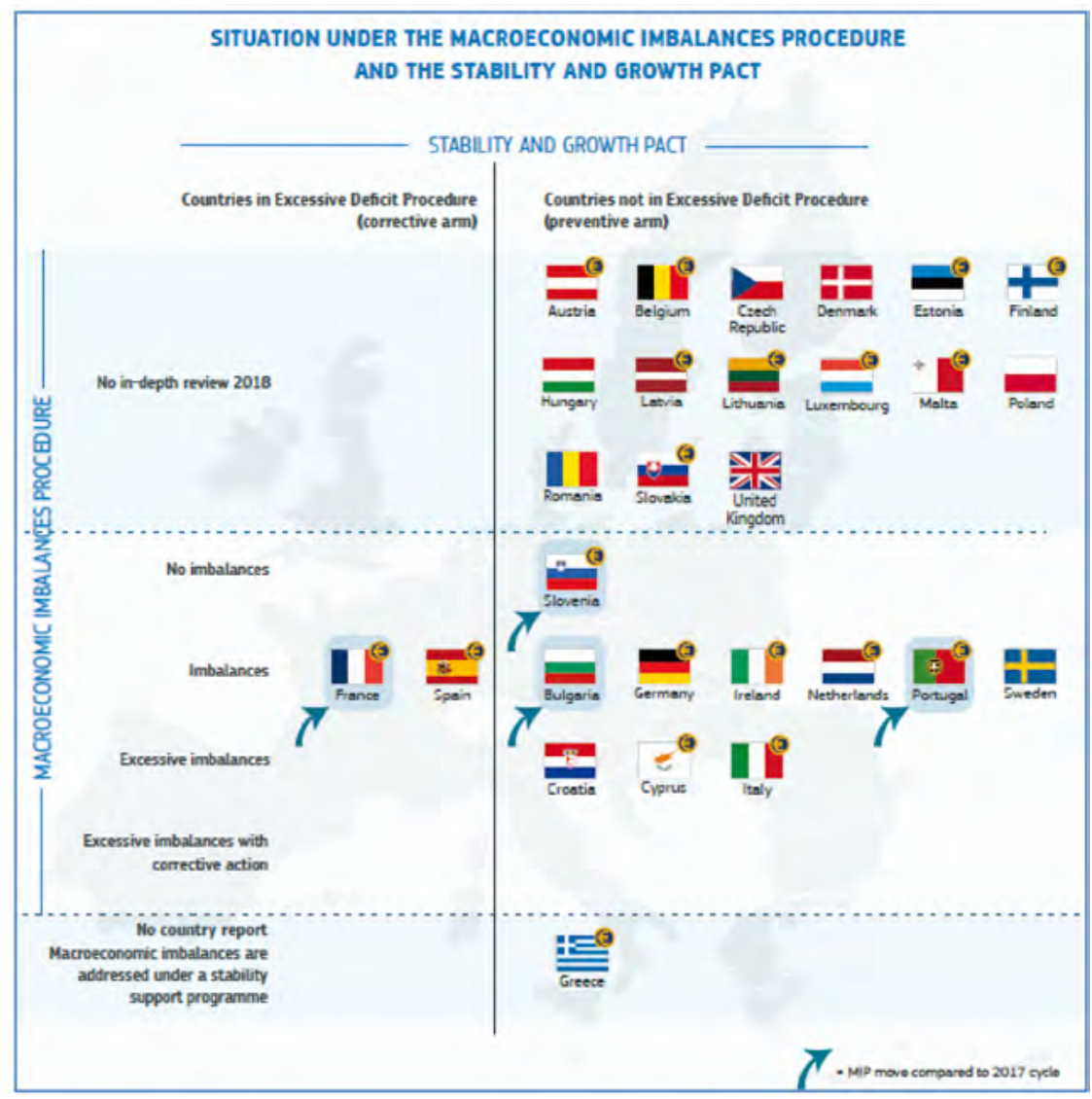

Semestre Europeo 2018 (actualización, marzo de 2018)

Fuente: Comisión Europea. 
Y en mayo la Comisión presentó su «paquete de primavera», con sus propuestas de Recomendaciones Específicas por país, REPs, que proporcionan una guía de políticas económicas (reformas presupuestarias, macroeconómicas y estructurales) para los próximos 12-18 meses, adaptadas a cada país con el fin de apoyar la creación de empleo y el crecimiento, y corregir desequilibrios detectados, garantizando el mantenimiento de unas finanzas públicas saneadas.

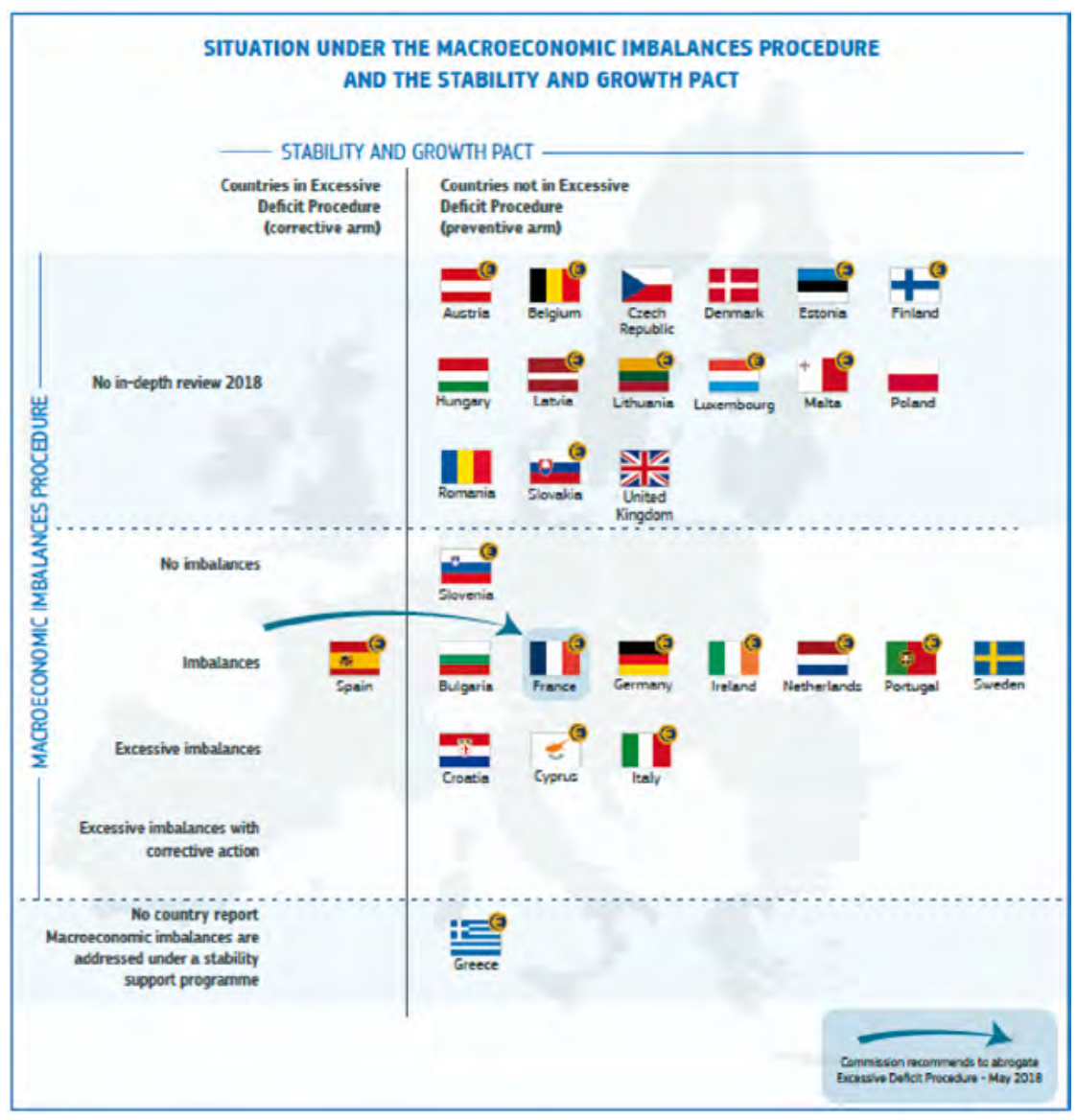

Semestre Europeo 2018 (actualización, mayo de 2018)

Fuente: Comisión Europea.

Según los servicios de análisis económico del ejecutivo comunitario, la mejora del contexto económico debería permitir centrar la atención en un 
nuevo conjunto de prioridades, teniendo en cuenta la estrecha interdependencia de las economías de la UE, y en especial de las de la zona del euro. La Comisión alentaba a los Estados miembros a emprender reformas estructurales que mejoren el entorno empresarial y las condiciones para la inversión, concretamente reformando los mercados de productos y servicios, apoyando la innovación, mejorando el acceso de las pequeñas y medianas empresas a la financiación y combatiendo la corrupción. Y alertaba también sobre la necesidad de que los Estados miembros refuercen su resiliencia económica ante retos a largo plazo como las tendencias demográficas, la emigración y el cambio climático, puesto que sólo unas Economías resistentes a largo plazo «pueden garantizar la convergencia económica y la reducción de las desigualdades».

En esta ocasión, Moscovici declaró que «hoy estamos más cerca de dejar atrás el legado de la crisis, ahora que Francia sale del procedimiento de déficit excesivo después de nueve años. Por primera vez desde la creación de la moneda única, en 2018 el déficit de todos los países de la zona del euro se situará por debajo del $3 \%$ del PIB. Guiar a los países de la UE a este punto nos ha llevado años de políticas presupuestarias responsables, y debemos garantizar que la responsabilidad siga siendo la clave también en el futuro».

En efecto, la Comisión recomendaba el cierre el procedimiento de déficit excesivo para Francia, con lo que únicamente un país, España, queda sometido al componente corrector del Pacto de Estabilidad y Crecimiento, frente a los 24 países que lo estaban en 2011.

\section{Competencia-Pacto fiscal Luxemburgo - Engie}

La Comisión Europea tomó una nueva decisión en junio sobre pactos fiscales ilegales contra Luxemburgo.

En esta ocasión, Bruselas decidió que la empresa Engie deberá devolver 120 millones de euros al Gran Ducado ya que, según la investigación iniciada en Septiembre de 2016, la compañía francesa de energía se habría beneficiado durante una década de un acuerdo para reducir su factura fiscal.

Engie, nacida de la fusión de Gaz de France y Suez, está participada por el Estado francés en un $24,1 \%$, aunque su poder real es mayor, dado que controla más de un tercio del derecho de voto.

La estructura financiera creada por la entidad, concebida para reducir artificialmente el pago de impuestos, hizo que en la práctica abonara tan solo un 0,3\% de sus beneficios en concepto de Impuesto de Sociedades, lo que según el criterio de Bruselas es una ayuda de Estado ilegal por incumplir las normas de la competencia y situar a Engie en una posición de privilegio respecto a sus rivales. 
«Luxemburgo ha proporcionado ventajas fiscales ilegales a Engie», explicó en rueda de prensa Margrethe Vestager, comisaria de Competencia, ratificando que la recuperación de ayudas de Estado ilegales en el plano tributario es una de sus principales líneas de actuación.

Luxemburgo mostró su total desacuerdo con la Comisión y emitió un comunicado en el que negaba haber proporcionado ayudas ilegales y afirmaba reservarse el derecho a recurrir la decisión. En todo caso, no es el primer caso en el que el Gran Ducado debe enfrentarse a este tipo de acusaciones, ya que Fiat y Amazon ya fueron obligadas a devolver impuestos a Luxemburgo en 2015 y 2017 respectivamente. La compañía automovilística eludió el pago de entre 20 y 30 millones, mientras que el gigante del comercio electrónico redujo ilegalmente su factura en 250 millones. Y otra multinacional, McDonald's también está siendo investigada por haberse beneficiado de ventajas fiscales en el país.

El caso más relevante por lo elevado de su importe ha sido, hasta la fecha, el de Apple, ya que la Comisión dictaminó en 2016 que la multinacional americana debía pagar a Irlanda 13.000 millones de euros en concepto de impuestos impagados, una suma sin precedentes. En abril, ambas partes anunciaron el acuerdo alcanzado para que la empresa estadounidense iniciara el desembolso de la suma establecida con una primera serie de pagos durante seis meses a partir del mes de mayo.

De hecho, el retraso en la ejecución del pago había llevado a la Comisión Europea a denunciar a Dublín ante la justicia europea en octubre de 2017, ante la paradoja de que el beneficiario de la decisión europea, Irlanda, fuese también quien más críticas vertió sobre la misma.

«El Gobierno está en desacuerdo con la resolución de la Comisión Europea. Sin embargo, como miembro comprometido de la UE, tenemos la intención de cumplir con nuestras obligaciones legales», señaló Paschal Donohoe, ministro irlandés de Finanzas.

\section{Sobre la autora}

Beatriz Iñarritu Ibarreche es Doctora por la Universidad de Deusto y Licenciada en Ciencias Económicas y Empresariales por la Universidad Comercial de Deusto. Su tesis, «Sistemas de Gobernanza Económica Europea: propuesta de Análisis y Medición», la realizó en el marco del programa de Estudios Internacionales e Interculturales de la Universidad de Deusto. Desde 1988, ha impartido docencia sobre Economía de la UE, en las Licenciaturas de Ciencias Económicas y Empresariales y Derecho de la UD, en la Escuela Universitaria de la Cámara de Comercio de Bilbao, y en los Institutos de Ocio y de Estudios Europeos de la UD. Actualmente, 
imparte docencia en el Departamento de Economía de Deusto Business School de la UD. Socia de Estrategias Empresariales Europeas, E-3, en la que dirige y desarrolla diferentes proyectos de consultoría en el campo de la internacionalización empresarial y de la UE. Ha sido profesora en el Diploma sobre Derecho Comunitario de la Universidad Iberoamericana de México en colaboración con la UD, impartiendo el módulo sobre «Mercado Interior Europeo e implantación del Euro» en México DF. Es ponente habitual en jornadas organizadas por entidades y organismos empresariales que contribuyen al mayor conocimiento de las diferentes cuestiones relacionadas con la UE y la internacionalización empresarial. Ha participado en un Equipo de Investigación de la UD en el proyecto «Instrumentos Innovadores de Gobernanza de la Unión Europea. Impacto en la gestión pública y en la competitividad regional».

Es autora de la sección «Crónica Comunitaria: la actualidad institucional y económica de España en el marco de la UE» en «Cuadernos Europeos de Deusto», revista editada por la UD. Es coautora de las publicaciones anuales «Guía de la Movilidad en la UE» y «Apuntes sobre la UE y Bizkaia» editadas por la Diputación Foral de Bizkaia. Ha sido coautora del Caso de Internacionalización publicado por el ICEX: «Inauxa, una Pyme en el Mercado Mundial de Automoción».

\section{About the author}

Beatriz Iñarritu Ibarreche is a Doctor of the University of Deusto and holds a degree in Economics and Business Studies from the Commercial University of Deusto. Her thesis, «Systems of European Economic Governance: Proposal for Analysis and Measurement», was carried out within the framework of the program of International and Intercultural Studies of the University of Deusto. Since 1988, she has lectured on EU Economics, in the Bachelor's of Economics and Business Studies and Law at the UD, at the University School of the Chamber of Commerce of Bilbao, and at the Institutes of Leisure and European Studies of the UD. Currently she teaches in the Department of Economics of Deusto Business School of the UD. Partner of European Business Strategies, E-3, in which she directs and develops different consulting projects in the field of business and EU internationalization. She has taught in the Diploma on Community Law of the Universidad Iberoamericana de México in collaboration with the UD, giving the module on «European Internal Market and implementation of the Euro» in Mexico DF. She is a regular speaker at conferences organized by business entities and organizations that contribute to a better understanding of the different issues related to the EU and business 
internationalization. She has participated in a Research Team of the UD in the project «Innovative Instruments of Governance of the European Union. Impact on public management and regional competitiveness».

She is the author of the section «Community Chronicle: the institutional and economic news of Spain within the framework of the EU» in «Cuadernos Europeos de Deusto», a magazine edited by the UD. She is co-author of the annual publications «Guide to Mobility in the EU» and «Notes on the EU and Bizkaia» edited by the Provincial Council of Bizkaia. She has coauthored the Internationalization Case published by the ICEX: «Inauxa, a SME in the World Automotive Market». 


\section{Derechos de autor}

Los derechos de autor (para la distribución, comunicación pública, reproducción e inclusión en bases de datos de indexación y repositorios institucionales) de esta publicación (Cuadernos Europeos de Deusto, CED) pertenecen a la editorial Universidad de Deusto. El acceso al contenido digital de cualquier número de Cuadernos Europeos de Deusto es gratuito inmediatamente después de su publicación. Los trabajos podrán leerse, descargarse, copiar y difundir en cualquier medio sin fines comerciales y según lo previsto por la ley; sin la previa autorización de la Editorial (Universidad de Deusto) o el autor. Así mismo, los trabajos editados en CED pueden ser publicados con posterioridad en otros medios o revistas, siempre que el autor indique con claridad y en la primera nota a pie de página que el trabajo se publicó por primera vez en $C E D$, con indicación del número, año, páginas y DOI (si procede). Cualquier otro uso de su contenido en cualquier medio o formato, ahora conocido o desarrollado en el futuro, requiere el permiso previo por escrito del titular de los derechos de autor.

\section{Copyright}

Copyright (for distribution, public communication, reproduction and inclusion in indexation databases and institutional repositories) of this publication (Cuadernos Europeos de Deusto, CED) belongs to the publisher University of Deusto. Access to the digital content of any Issue of Cuadernos Europeos de Deusto is free upon its publication. The content can be read, downloaded, copied, and distributed freely in any medium only for non-commercial purposes and in accordance with any applicable copyright legislation, without prior permission from the copyright holder (University of Deusto) or the author. Thus, the content of $C E D$ can be subsequently published in other media or journals, as long as the author clearly indicates in the first footnote that the work was published in $C E D$ for the first time, indicating the Issue number, year, pages, and DOI (if applicable). Any other use of its content in any medium or format, now known or developed in the future, requires prior written permission of the copyright holder. 